\title{
Instability of Glycemia before Centrifugation without Antiglycolytic and Correction Factors of Differed Glycemia Assays
}

\author{
Bianza Moise Bakadia1 ${ }^{*}$, Tshilumbayi Falone Mutoba1, Bakamona Lina Babidi², \\ Kabengele Arlette Ndaya ${ }^{1}$, Mulongo Pauline Kalenga ${ }^{1}$, Kamona Charles Kimuni ${ }^{1}$, \\ Pembi Christian Kasongo ${ }^{1}$, Lusenge Nono Ndete ${ }^{1}$, Mutumukole Jeanluc Biampata ${ }^{3}$, \\ Ndibualonji Bualufubadibanga Victor ${ }^{4}$
}

\author{
${ }^{1}$ Department of Medical Biology, Higher Institute of Medical Techniques of Lubumbashi, Lubumbashi, DR Congo \\ ${ }^{2}$ Department of Nursing Sciences, Higher Institute of Medical Techniques of Lubumbashi, Lubumbashi, DR Congo \\ ${ }^{3}$ School of Public Health, University of Lubumbashi, Lubumbashi, DR Congo \\ ${ }^{4}$ University of Lubumbashi, Faculty of Veterinary Medicine, Lubumbashi, DR Congo \\ Email: ^bakadiafalone @ gmail.com
}

How to cite this paper: Bakadia, B.M., Mutoba, T.F., Babidi, B.L., Ndaya, K.A., Kalenga, M.P., Kimuni, K.C., Kasongo, P.C., Ndete, L.N., Biampata, M.J. and Victor, N.B. (2018) Instability of Glycemia before Centrifugation without Antiglycolytic and Correction Factors of Differed Glycemia Assays. Open Access Library Journal, 5: e4783.

https://doi.org/10.4236/oalib.1104783

Received: July 16, 2018

Accepted: August 13, 2018

Published: August 16, 2018

Copyright $\odot 2018$ by authors and Open Access Library Inc.

This work is licensed under the Creative Commons Attribution International License (CC BY 4.0).

http://creativecommons.org/licenses/by/4.0/ (c) (i) Open Access

\begin{abstract}
Insert Glycolysis that occurs in vitro venous blood samples is known to induce a rapid drop in blood sugar. The use of an antiglycolytic is problematic, sodium fluoride creates hemolysis and mono-iodoacetate is active two hours after sampling. However, many laboratories are uncentrifuged specimens from distant sampling sites with varying delivery times sometimes several hours. The objective of this study is firstly to determine the influence of glycolysis on deferred blood glucose assays and secondly to develop correction factors of deferred assays blood glucose. The study was conducted on 30 volunteers supposed saints. Four tubes $7 \mathrm{ml}$ have been collected by volunteer: Two tubes with lithium heparin (TH) and two tubes without anticoagulant (TS). For 30 consecutive days, a different volunteer was taken daily at the same time. A tube without anticoagulant and TH tube were immediately centrifuged to determine the glucose reference or blood glucose $T_{0}$. The remaining two tubes, $\mathrm{TH}$ and TS were respectively divided into three aliquots into tubes eppendorf. At each time $(t): 1 \mathrm{~h}, 2 \mathrm{~h}, 3 \mathrm{~h}$, an aliquot of each tube TH and TS was centrifuged prior to the determination of blood glucose. The analysis results show a comparable decrease in blood glucose of about $10 \%$ per hour for both types of tubing. The regression $Y=1.08 X+2.5$ has been established between the values of glucose $T_{0}$ and $T_{1}$ values of blood glucose, $Y$ is the value of blood glucose $T_{0}$ and $X$ is the value of the $T_{1}$ glucose, the right regression $Y=1.21 X+1.6$ was found between blood glucose values $T_{0}$ and
\end{abstract}


blood glucose values $T_{2}, Y$ is the value of blood glucose $T_{0}$ and $X$ is the value of blood glucose $T_{2}$. The regression $Y=1.32 X+2.5$ has been established between the values of glucose $T_{0}$ and $T_{3}$ values of blood glucose, $Y$ is the value of blood glucose $T_{0}$ and $X$ is the value of the $T_{3}$ glucose. The correlation coefficient $r=0.99$ shows a positive correlation between $X$ and $Y$, which is directly proportional. The established straight linear regression is likely to be used as a corrective factor by health professionals when blood glucose has not been measured directly after sampling or when separation of serum or plasma and blood elements is not immediate.

\section{Subject Areas}

Bioengineering

\section{Keywords}

Glycemia, Antiglycolytic, Correction Factor, Linear Regression Line

\section{Introduction}

To ensure its function of transporting hemoglobin and maintaining its existence, the red blood cell must constantly fight against two dangers; oxidation of its constituents; iron, hemoglobin and membrane proteins and its own hyperhydration. The means of this fight are provided by intra-erythrocyte glycolysis. This physiology explains the high consumption of glucose by red blood cells which is why blood is taken from a glycolysis-inhibiting anticoagulant when plasma glucose is to be determined [1]. Diabetes screening and diagnosis, a major public health problem are based on threshold values for blood glucose. The determination of blood glucose is thus one of the most frequently performed analyses in hospitals as in city laboratories. If the analytical phase is perfectly controlled, some steps of the pre-analysis phase, especially the transmission delay, are less standardized and therefore condition the quality of the results. There is evidence of a lack of serum glucose stability or serum plasma stability [2] [3]. They are mostly old and have little evidence of stability in whole blood with or without antiglycolics. However, many laboratories are receiving non-centrifuged specimens from remote collection sites with varying delivery times, sometimes several hours. To overcome this problem, manufacturers of tubes for the collection of biological samples have proposed for several years the use of antiglycolytic agents, which are supposed to guarantee the stability of this parameter over time [4]. The most frequently used antiglycolytics are sodium fluoride and monoiodoacetate but unfortunately sodium fluoride creates hemolysis and the anti-glycolytic action of mono-iodoacetate is noticeable beyond two hours [5]. As part of the laboratory quality approach, and in particular the reflection conducted on the preanalytical step, we were led to study the instability of glycemia on lithium heparin tube and tube without anticoagulant, to determine the influ- 
ence of the glycolysis which occurs in vitro on the blood samples and to develop the correction factors of the delayed glycemic dosages compared to the blood glucose measurements directly after sampling.

\section{Materials and Methods}

This is a prospective and analytical study, conducted in Lubumbashi (DR Congo) and spread over one month (March 2017). The experimental setting was the laboratory of applications of Higher Institute of Medical Techniques of Lubumbashi. The study was conducted on 30 volunteers, 13 women and 17 men, apparently healthy, aged 19 to 44, after obtaining free and informed consent. Venous blood was collected according to good sampling practices in each of 30 subjects, fasting for 8 hours and seated, by venipuncture antecubital. Four tubes of 7 $\mathrm{ml}$ were thus collected by volunteer: two tubes with lithium heparin $(\mathrm{TH})$, commonly used for the determination of many parameters including blood glucose and two tubes without anticoagulant (TS). For 30 consecutive working days, a different volunteer was taken daily at the same time. A tube without anticoagulant and a TH tube were immediately centrifuged for 10 minutes at 1500 $\mathrm{g}$ at room temperature $\left(25^{\circ} \mathrm{C}\right)$ to determine the reference blood glucose level. The two remaining $\mathrm{TH}$ and TS were respectively fractionated into three aliquots in eppendorff tubes immediately stored in a thermostated chamber at $20^{\circ} \mathrm{C}$ $\left( \pm 1^{\circ} \mathrm{C}\right)$, protected from light. At each of the following times $(\mathrm{t}): 1 \mathrm{~h}, 2 \mathrm{~h}, 3 \mathrm{~h}$, an aliquot of each of TH and TS tubes was centrifuged for $10 \mathrm{~min}$ at $1500 \mathrm{~g}$ prior to determination of the blood glucose. A qualitative evaluation of the appearance of plasma and serum has also been performed in order to eliminate any macroscopic interference (hemolysis, turbidity). The study was carried out on the $\mathrm{BD}$ Vacutainer TM lithium heparin (TH) tubes and test tube without anticoagulant from cypress diagnostics. This choice was motivated by their use in daily practice in different laboratories. The glycemic determination was performed on cyanplus spectrophotometer (Cypress Diagnostics) by glucose oxidase technique. For statistical analysis, we used the hypothesis test and meanings that is to say, we realized the distribution of the difference of the means between the values of the blood glucose $T_{0}$ and the blood glucose $t_{1}, t_{2}, t_{3}$ for the tubes TH and TS at probability threshold of $5 \%$. To determine the deferred glycemic correction factors, we used the statistical series adjustment test, i.e. we computed the linear regression line equations, the correlation and the determination coefficients between $T_{0}$ and $T_{1}, T_{2}, T_{3}$ blood glucose. The excel 2003 software was used for data processing in the form of plot lines and point clouds. All procedures herein were approved by the Research Ethics Committee of the Higher Institute of Medical Techniques of Lubumbashi, with participants giving informed consents before entering the study.

\section{Results}

No visible interference has been demonstrated by the qualitative study of plasma 
and serum at different times. The blood glucose values in $\mathrm{mg} / \mathrm{dl}$ are presented in Table 1, they vary at $T_{0}$ for the TH tube between 64 and 123 and for the TS tube between 65 and 125 and at $T_{3}$ for the TH tube between 46 and 89 and for the TS tube between 47 and 91 . The statistical analysis does not show any significant

Table 1. Blood glucose values $(\mathrm{mgl} / \mathrm{dl})$ at different times for each $\mathrm{TH}$ and TS tube.

\begin{tabular}{|c|c|c|c|c|c|c|c|c|}
\hline \multirow[b]{2}{*}{ Time } & \multicolumn{4}{|c|}{ Heparinate tube Lithium (th) } & \multicolumn{4}{|c|}{ Tube without anticoagulant (TS) } \\
\hline & 0.0 & $1 \mathrm{~h}$ & $2 \mathrm{~h}$ & $3 \mathrm{~h}$ & 0.0 & $1 \mathrm{~h}$ & $2 \mathrm{~h}$ & $3 \mathrm{~h}$ \\
\hline \multicolumn{9}{|c|}{ Subjetcs } \\
\hline 1 & 84 & 76 & 68 & 61 & 85 & 77 & 69 & 62 \\
\hline 2 & 118 & 106 & 95 & 86 & 120 & 108 & 97 & 87 \\
\hline 3 & 80 & 71 & 64 & 57 & 80 & 72 & 65 & 58 \\
\hline 4 & 95 & 84 & 77 & 70 & 96 & 86 & 78 & 70 \\
\hline 5 & 87 & 78 & 71 & 63 & 88 & 79 & 71 & 64 \\
\hline 6 & 76 & 67 & 60 & 54 & 76 & 68 & 62 & 55 \\
\hline 7 & 85 & 77 & 70 & 63 & 87 & 78 & 70 & 63 \\
\hline 8 & 109 & 99 & 87 & 79 & 110 & 99 & 89 & 80 \\
\hline 9 & 77 & 69 & 62 & 57 & 78 & 70 & 63 & 57 \\
\hline 10 & 89 & 80 & 72 & 65 & 89 & 80 & 72 & 65 \\
\hline 11 & 93 & 83 & 75 & 68 & 94 & 84 & 76 & 69 \\
\hline 12 & 121 & 110 & 99 & 88 & 123 & 111 & 100 & 90 \\
\hline 13 & 87 & 78 & 70 & 63 & 87 & 78 & 70 & 63 \\
\hline 14 & 77 & 68 & 61 & 55 & 77 & 69 & 62 & 56 \\
\hline 15 & 85 & 76 & 69 & 62 & 86 & 77 & 70 & 63 \\
\hline 16 & 80 & 71 & 64 & 57 & 80 & 72 & 64 & 58 \\
\hline 17 & 69 & 60 & 55 & 49 & 69 & 62 & 56 & 50 \\
\hline 18 & 88 & 78 & 70 & 53 & 88 & 79 & 71 & 64 \\
\hline 19 & 123 & 111 & 100 & 90 & 125 & 113 & 101 & 91 \\
\hline 20 & 97 & 87 & 77 & 70 & 98 & 88 & 79 & 71 \\
\hline 21 & 90 & 80 & 72 & 65 & 90 & 81 & 73 & 66 \\
\hline 22 & 93 & 83 & 74 & 66 & 93 & 84 & 75 & 68 \\
\hline 23 & 108 & 98 & 88 & 79 & 110 & 99 & 89 & 80 \\
\hline 24 & 75 & 67 & 61 & 54 & 76 & 68 & 62 & 55 \\
\hline 25 & 91 & 84 & 73 & 57 & 93 & 84 & 75 & 68 \\
\hline 26 & 69 & 62 & 56 & 50 & 69 & 62 & 56 & 50 \\
\hline 27 & 78 & 69 & 61 & 56 & 78 & 70 & 63 & 57 \\
\hline 28 & 65 & 59 & 52 & 47 & 65 & 59 & 53 & 47 \\
\hline 29 & 77 & 68 & 62 & 55 & 78 & 70 & 63 & 57 \\
\hline 30 & 100 & 90 & 81 & 73 & 101 & 91 & 82 & 74 \\
\hline
\end{tabular}


difference between the blood glucose values taken on the TH tube and the TS, however the difference of the means is significant at the probability threshold of $5 \%$ between the blood glucose values $T_{0}$ and the blood glucose values $T_{1}, T_{2}, T_{3}$. The equations of the regression lines, correlation and determination coefficients are presented in Tables 2-4. As the difference is not significant between TH and TS blood glucose values, these regression coefficients are applicable for both types of sampling.

From Table 2 it appears that the values of the blood glucose $T_{0}$ is explained at $98 \%$ by the line $Y=1.08 X+2.5 ; Y$ is the value of the blood glucose $T_{0}$ and $X$ is the value of the blood glucose $T_{1}$. The coefficient of correlation $r=0.99$ shows a positive correlation between $X$ and $Y$; which are directly proportional.

From Table 3 it appears that the values of the blood glucose $T_{0}$ is explained at $98 \%$ by the line $Y=1.21 X+1.6 ; Y$ is the value of the blood glucose $T_{0}$ and $X$ is the value of the blood glucose $T_{2}$. The correlation coefficient $r=0.99$ shows a positive correlation between $X$ and $Y$; which are directly proportional.

From Table 4 it appears that the values of the blood glucose $t_{0}$ is explained at $98 \%$ by the line $Y=1.32 X+3.5 ; Y$ is the value of the blood glucose $t_{0}$ and $X$ is the value of the blood glucose $t_{3}$. The correlation coefficient $r=0.99$ shows a positive correlation between $X$ and $Y$; which are directly proportional (Figure 1 and Figure 2).

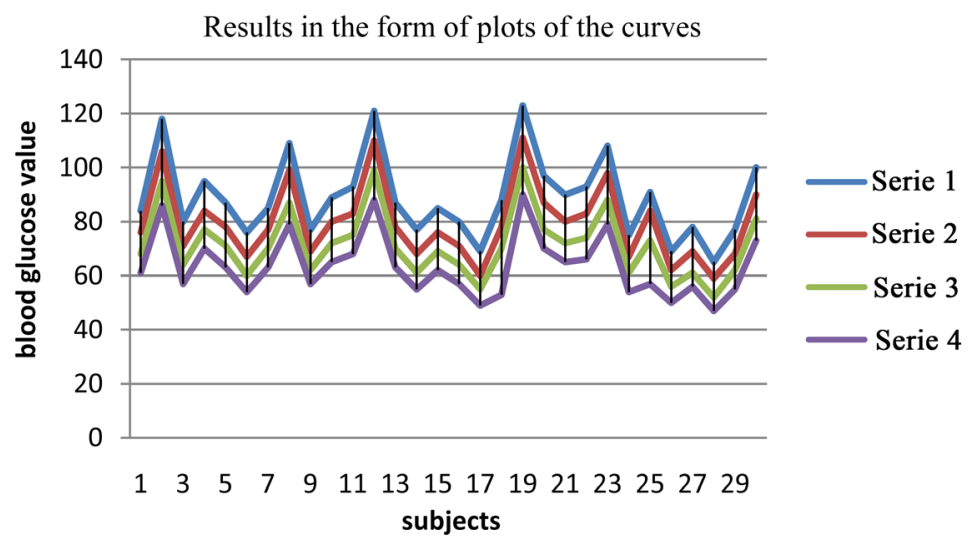

Figure 1. Presentation of the results in the form of plots of the curves. Série 1: blood glucose values $T_{0}$, Série 2: blood glucose values $T_{1}$, Série 3: blood glucose values $T_{2}$, Série 4: blood glucose values $T_{3}$. The plots of the curves of the results show that all the concentrations of the blood glucose $T_{0}$ are higher compared to the concentrations of delayed assays of blood glucose; $T_{1}, T_{2}, T_{3}$.

Table 2. Linear regression line, coefficient of correlation and coefficient of determination between $T_{0}$ blood glucose and $T_{1}$ blood glucose.

\begin{tabular}{cc}
\hline Parameters & Blood glucose $T_{0}(Y)$-Blood glucose $T_{1}(X)$ \\
\hline Linear regression line & $Y=1.08 X+2.5$ \\
Coefficient of correlation & $r=0.99$ \\
Coefficient of determination & $r^{2}=98 \%$ \\
\hline
\end{tabular}




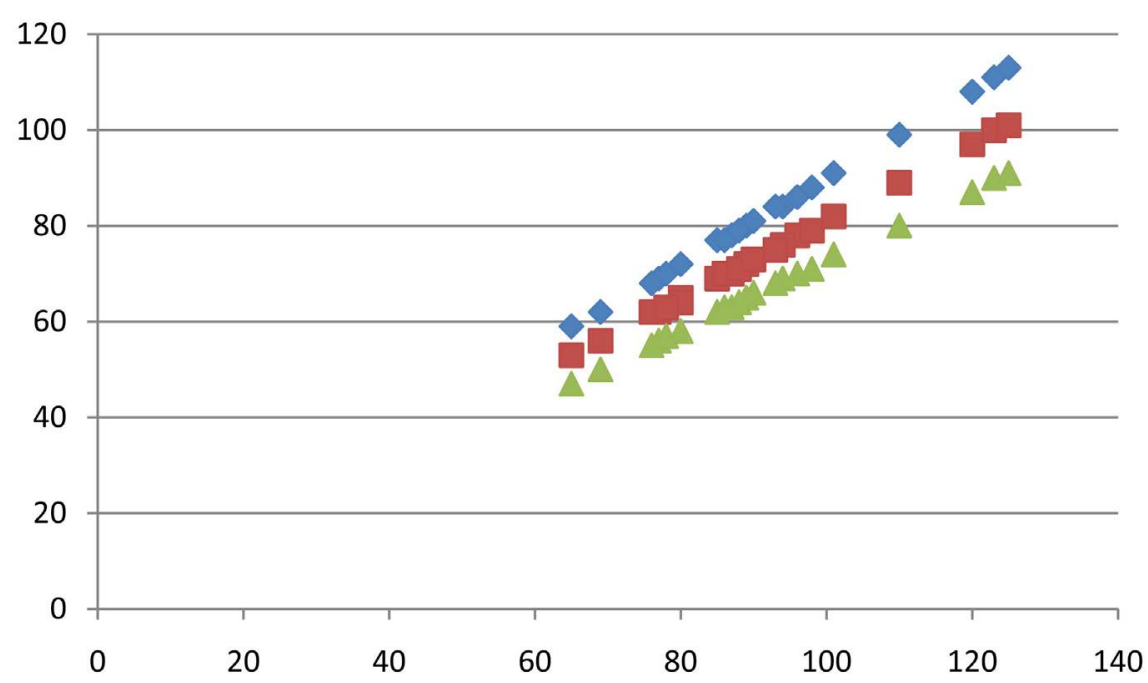

Figure 2. Presentation of the results in the form of point clouds. Herein shows that all the points tend to approach on the same lines, i.e. the correlation between the concentrations of the blood glucose $T_{0}$ and the blood glucose $T_{1}, T_{2}, T_{3}$ is positive linear.

Table 3. Linear regression line, correlation coefficient and coefficient of determination between blood glucose $T_{0}$ and blood glucose $T_{2}$.

\begin{tabular}{cc}
\hline Parameters & Blood glucose $T_{0}(Y)$-Blood glucose $T_{2}(X)$ \\
\hline Linear regression line & $Y=1.21 X+1.6$ \\
Coefficient of correlation & $r=0.99$ \\
Coefficient of determination & $r^{2}=98 \%$ \\
\hline
\end{tabular}

Table 4. Linear regression line, coefficient of correlation and coefficient of determination between $T_{0}$ blood glucose and $T_{3}$ blood glucose.

\begin{tabular}{cc}
\hline Parameters & Blood glucose $T_{0}(Y)$-Blood glucose $T_{3}(X)$ \\
\hline Linear regression line & $Y=1.32 X+3.5$ \\
Coefficient of correlation & $r=0.99$ \\
Coefficient of determination & $r^{2}=98 \%$ \\
\hline
\end{tabular}

\section{Discussion}

The glycolysis that occurs in vitro on the venous blood sample is known to cause a rapid decline in blood glucose. The use of an antiglycolytic agent is supposed to guarantee the stability of this parameter over time, but unfortunately antiglycolytics are problematic. A study carried out by B. Foucher et al. shows that it is only after the second hour that the anti-glycolytic effect of mono-iodoacetate is perceptible and the antiglycolytic agent; sodium fluoride creates hemolysis; this is a real problem for the screening of diabetic subjects whose measured blood glucose is close to the decision threshold when the centrifugation is not immediate [5]. Diagnosis criteria for diabetes mellitus are based on a fasting glucose level $\geq 11.1 \mathrm{mmol} / \mathrm{l}$ regardless of the time of day. Orally induced hyperglycemia 
(OIHG) remains the gold standard in the definition of diabetes (blood glucose greater than $11 \mathrm{mmol} / \mathrm{l}, 2$ hours after OIHG) but is no longer recommended by Anaes because of its heaviness and lack of reproducibility in the same patient [6]. The recommended test for diabetes screening is fasting blood glucose [7]. Diabetes screening and diagnosis are based on a specific biological assay and threshold values. Any changes in blood glucose attributable to preanalytical delay therefore hamper interpretation especially at values close to these decision thresholds. In vitro, glucose is metabolized following the cascade of glycolysis; the addition of an anti-glycolytic agent (mono-iodoacetate, sodium fluoride or potassium oxalate) must stop this phenomenon. Our study shows a significant difference between $T_{0}$ and $T_{1}, T_{2}, T_{3}$ blood glucose concentrations and a decrease in blood glucose levels of glycemia on the TH and TS tube by about $10 \%$ per hour this means that in vitro-induced glycolysis by red blood cell affects delayed blood glucose dosing, which is why the search for correction factors in the form of regression lines linear is of great importance. The regression line $y=1.08 X+$ 2.5 was established between the blood glucose values $T_{0}$ and the glucose values $T_{1}, y$ being the blood glucose value $T_{0}$ and $X$ is the blood glucose value $T_{1}$; the regression line $y=1.21 X+1.6$ has been established between the blood glucose values $T_{0}$ and the blood glucose values $T_{2}$, where $y$ is the blood glucose value $t_{0}$ and $X$ is the blood glucose value $t_{2}$; the regression line $y=1.32 X+3.5$ has been established between the blood glucose values $t_{0}$ and the glucose values $t_{3}$, where $y$ is the blood glucose value $t_{0}$ and $X$ is the blood glucose value $t_{3}$. All these lines were found with a correlation coefficient of $0.99 \%$, which explains a correlation between the two variables. This coefficient being greater than 0 , this means that these two variables are directly proportional. The values of blood glucose measured at time $t=0$ is explained at $98 \%$ as coefficient of determination by the different regression lines that is to say that we are $98 \%$ sure to have made a good correction in using the linear regression line when blood glucose was not measured directly after sampling.

\section{Conclusion}

The established linear regression lines; $y=1.08 X+2.5$ between the blood glucose values $T_{0}$ and the blood glucose values $T_{1}$, y is the blood glucose value $T_{0}$ and $X$ is the blood glucose value $T_{1}$; the regression line $y=1.21 X+1.16$ between the glucose values $T_{0}$ and the blood glucose values $T_{2}, y$ is the blood glucose value $T_{0}$ and $X$ is the blood glucose value $T_{2}$; the regression line $y=1.32 X+3.5$ between $T_{0}$ blood glucose values and $T_{3}$ blood glucose values are likely to be used as corrective factors by health professionals when blood glucose has not been measured directly after sampling or when separation serum or plasma and figured elements of blood is not immediate. Whatever the type of tube used, the attitude to be adopted is to inform the time of sampling to allow the biologist to convert the concentrations of delayed dosing of blood glucose using established linear regression lines. 


\section{Conflicts of Interest}

Undeclared.

\section{Authors' Contribution}

All authors contributed identically to the different phases of the research.

\section{References}

[1] Hennen, G. (1996) Biochimie humaine: Introduction biochimique à la médecine interne: De Boeck Supérieur.

[2] Zhang, D.J., Elswick, R., Miller, W.G. and Bailey, J.L. (1998) Effect of Serum-Clot Contact Time on Clinical Chemistry Laboratory Results. Clinical Chemistry, 44, $1325-1333$.

[3] Boyanton, B.L. and Blick, K.E. (2002) Stability Studies of Twenty-Four Analytes in Human Plasma and Serum. Clinical Chemistry, 48, 2242-2247.

[4] Vassault, A., Azzedine, M., Bailly, M., Cam, G., Dumont, G., Ekindjian, O., et al. (1986) Commission "Validation de techniques" (Commission on "Validation of Techniques"). Annales de Biologie Clinique, 44, 679-685.

[5] Foucher, B., Pina, G., Desjeux, G., Cheminel, V. and Prevosto, J. (2004) Stabilité de la glycémie avant centrifugation avec ou sans antiglycolytique. Annales de Biologie Clinique, 601-604.

[6] Fonfrede, M. (2004) Diabète de type 2: Les bons outils du diagnostic et du suivi en 2003. Spectra Biologie, 23, 35-39.

[7] Chevillon, I., Larrose, C., Moreau, N. and Orsonneau, J.-L. (1998) Conservation des échantillons de sang avant analyse des paramètres biochimiques les plus courants. Annales de Biologie Clinique, 200-204.

\section{Abbreviations Used}

Tube TH: tube containing lithium heparin, Tube TS: tube without anticoagulant, blood glucose $T_{0}$ : blood glucose measured directly after sampling, blood glucose $T_{1}$ : blood glucose measured one hour after sampling, blood glucose $T_{2}$ : blood glucose measured two hours after sampling, blood glucose $T_{3}$ : blood glucose measured three hours after sampling. 